\title{
New automatic quality control methods for geometrical treatment planning system tools in external conformal radiotherapy
}

\author{
Eloïse Denis ${ }^{a}$, Stéphane Beaumont ${ }^{b}$, JeanPierre Guédon ${ }^{a}$, \\ Tarraf Torfeh ${ }^{a}$, Nicolas Normand ${ }^{a}$, Norbert Ailleres ${ }^{c}$
}

\begin{abstract}
Quality control of external conformal radiotherapy treatment planning systems softwares is a crucial issue. The treatment quality depends directly on the quality of treatment planning systems (TPS). Radiotherapists need to be sure that softwares compute accurately each parameter of the treatment. This paper focuses on the quality control of geometrical tools of the treatment planning systems, i.e. the virtual simulation software. These TPS compute the geometrical part of the treatment. They define the targets and shapes of the irradiation beams. Four operations done by these TPS are examined in this work. The quality control of the auto-contouring, auto-margin, isocenter computation and collimator conformation tools is treated with a new method based on Digital Test Objects (DTO). Standard methods for this quality control have been set up from the development of some Physical Test Objects (PTO). ${ }^{1,2}$ These methods are time-consuming, incomplete and inaccurate. Results are biased by the CT-scanner acquisition of PTOs and error evaluation is done with the graphic tools of the TPS. Our method uses DTOs and allows for an automated qualitative error evaluation. DTOs present many advantages for TPS quality control. ${ }^{3-5}$ They lead to a fast, accurate, complete and automatic quality assessment. Special DTOs have been developed to control the TPS tools mentioned previously as well as their automatic result analysis methods. A TPS has been controlled with these test objects. The quality assessment shows some errors and highlights some particularities in the TPS tools functioning. This quality control was then compared with the standard quality control.
\end{abstract}

Keywords: Quality control, treatment planning system, radiotherapy, virtual simulation, CT-simulation, digital test object, digital phantom, physical test object, phantom

\section{INTRODUCTION}

This paper focuses on the quality control of the geometrical part of the Treatment Planning Systems (TPS) i.e. virtual simulation software for external radiotherapy. The main functions of this geometric simulation software are the automatic organ contouring, automatic expansion of the target volume, automatic isocenter localization and collimator conformation. These four steps, contribute to determine the accuracy and consequently the effectiveness of the treatment by ensuring the delivery of the X-ray dose on all the tumor and preserving the healthy tissues near the tumor. The TPS quality control is traditionally done using Physical Test Objects (PTO) aligned in the CT-scanner. The PTO images are taken down indirectly on the TPS and compared with geometrical characteristics of the PTO. This quality control method is not optimal: the alignment of the object and acquisition of the PTO with the CT-scanner is very time-consuming and source of inaccuracies. CT-scanner is monopolized during all the quality control leading to only partial accomplishment. Moreover, physical test objects are very expensive to manufacture. It is also difficult to modify and adapt their design to follow the legislation or the evolution of the controlled software. And first of all, the CT acquisition of the physical test objects distorts its reality and disturbs the accuracy of the TPS quality control. This paper proposes a new

\footnotetext{
Further author information: (Send correspondence to Eloïse Denis)

${ }^{a}$ IRCCyN/IVC - UMR CNRS 6597, Image \& Video Communication, Nantes - France

Email: \{eloise.denis, jean-pierre.guedon, tarraf.torfeh , nicolas.normand\}@polytech.univ-nantes.fr

${ }^{b}$ QualiFormeD, La Roche Sur Yon - France

Email: stephane.beaumont@qualiformed.com

${ }^{c}$ Centre Régional de Lutte Contre le Cancer, Val d'Aurelle, 34298 Montpellier - France
} 
method to control the quality of these geometrical parts of the TPS with the use of Digital Test Objects (DTO). Such objects are used to make the quality control automatic. They avoid alignment and acquisition problems leading the control more precise and accurate. Associated with automatic analysis method, they allow for an objective quantitative error assessment. Moreover, DTOs are easier to manufacture than PTO and can be modified easily to be adjustable and progressive.

\section{CLASSICAL QUALITY CONTROL METHODS}

Quality control of radiotherapy treatment planning systems is now mandatory in every hospital. ${ }^{6-9}$ It ensures a good treatment parameters computation to target accurately all the tumor with a minimum irradiation of the neighboring healthy organs. Standard methods are based on the use of physical test objects (PTOs or phantoms). We present here the major PTOs used for the TPS geometric tools quality assessment.

The American Association of Physicists (AAPM) recommends in its task groups $\mathrm{n}^{\circ} 53^{6}$ to check the correctness of the geometric tools algorithms, and adds in its task group $n^{\circ} 66^{7}$ to use the geometric part of the Craig phantom ${ }^{1}$ which commercial version is distributed by Modus. ${ }^{10}$ Craig's physical test objects are made up of two volumes and allows for a quality control for the whole TPS. The first one is made up of a divergence shape put on two degrees of freedom rotation system which simulate gantry and table rotation of the linac, i.e. beam incidence. It is used to control all the geometrical part of the beams definition: the beam divergence and incidence, the isocenter positioning, the conformation of the collimator (with or without multi-leaf system) and the digital reconstructed radiographs (DRR) computation. The second one is made of an acrylic oval shape with cylindrical holes to put a specific object for each quality test. This volume allows for controlling the precision of $2 \mathrm{D}$ and $3 \mathrm{D}$ reconstruction, the tumor contouring, the auto-margin tools to expand the target, the dose volume histograms and the conversion of CT numbers to relative electron densities.

The first object is thus used to control the collimator parameterization. It is made up of a divergent volume with a cross shaped section. This volume dimensions correspond to several multi-leaf collimators specifications that makes possible to conform the collimator exactly to the shape. Positioning the PTO in several incidences, it is possible to check if the TPS computes the right parameters for the collimator. This object can be used in the same way for a traditional collimator. The second object is used to control the targeting of the tumor by the TPS. Different shapes as cubes, cylinders and spheres of different densities can be inserted in the object. The quality control consists to make an automatic targeting in the TPS and seeing on the screen if the computed region of interest (ROI) fits well with the tested shape. The quality control of auto-margin algorithms using the physical test object is done with the same devices. Some expansions are computed from a ROI including the shapes and the characteristics of the expansions can be seen on the TPS screen. It is then possible to compare the response of different TPSs to the same expansion test of a particular shape.

Tenon hospital of Paris (France) developed and patented its own phantom. ${ }^{2}$ Among a lot of functionalities for DRR quality, it allows for controlling the auto-contouring, auto-margin and isocenter positioning TPS tools. Auto-contouring verification is done with a polystyrene cube and two cylinders of different densities. The error evaluation is assessed comparing the surface of the PTO to the surface of the resulted structure, computed by the TPS. The auto-margin tool is controlled using a cone with two different concentric densities. Along an axis, margins between the two cones are uniform and non uniform along the perpendicular axis. The error evaluation is done using the same technique as for auto-contouring verification. Isocenter positioning is checked with a cube including a central bead, marked on the faces to be aligned with the lasers. The error is assessed by verifying if the computed isocenter coordinates correspond to the laser marks on the TPS display.

In his PhD thesis, Georges Madelis ${ }^{11}$ suggested a quality control method for auto-margin algorithms based not on shapes phantoms but on digital test objects defined by discretized shapes for radiotherapy. A panel of several objects is designed to cover all the requirements for a complete quality test. Uniform and non uniform margins are tested on cubic, cylindrical and concave regions of interest. He defined moreover some test objects to analyze the way the TPS takes the slice thickness in the expansion computation and to check the avoidance of an organ. His approach is then to base the expansion quality control not on a TPS computed region of interest but on a perfect and well known structure. With this method, the expansion test can be very accurate. Only auto-margin is tested and the targeting does not interfere with the control as it is the case with the physical test object used. 


\section{MATERIAL}

In this work, new digital test objects are presented for the geometric quality control of a TPS. These test objects are made up of transversal slices of geometric shapes, beams and structures information. In order to create them, a dedicated software tool has been developed. This tool - named DTO-Creator - allows for creating almost any kind of geometrical volume, beam and structure objects adapted to each quality control requirement.

The main part of a digital test object is firstly a pure volume description, and then a discrete volume is stored in slices as some CT data of a physical object. However, DTOs have the major advantage to keep a perfect geometry corresponding to their shapes and densities definition as they are not deteriorated by their acquisition and possible non alignment in the CT-scanner. All shapes contained into the DTO volume are defined on the software in a continuous way from some primitive shapes and operations of these shapes. The available primitive shapes are associated with some sampling methods insuring equivalence or mastered non equivalence between the analytical model and the discrete one. ${ }^{4}$ The recording of the continuous description of the test object allows for an easy modification of the object geometry or density from its tree organization description. This image part of the digital test object can be completed by some files containing information about treatment beam and structures (regions of interest). DTO-Creator allows for the definition of some beams with gantry, couch and collimator angles, collimator conformation and almost any treatment parameters. It is moreover possible to define the contours of regions of interest (radiotherapy structures) corresponding to the target for the irradiation beams. All these DTO files can then be transferred to the controlled TPS in DICOM 3.0 and DICOM-RT format. ${ }^{12}$ Finally DTO-Creator software acts as a kind of inverted TPS. Actually, a TPS produces a virtual continuous model of the patient with 2D CT-scanner slices, and DTO-Creator produces 2D CT-scanner slices from a continuous geometry of a virtual phantom.

\section{METHODS}

We developed a quality control method based on digital test objects, specially designed for each quality criterion. Furthermore we set up a general method for the different geometrical TPS tools. Moreover, we defined some concepts in order to formalize it.

\subsection{General DTO method: input and output DTOs}

The quality control test object presented in this paper fits to a general method defined for all TPS quality control based on digital test objects. This method consists in replacing PTO CT-scanner images by digital test object perfect images. At the same time, output digital test objects are computed with some reference algorithms in order to serve as standards to characterize the TPS performances. They correspond to the theoretical response of a perfect TPS. These reference output objects are compared to the TPS results of the quality test, themselves considered as DTOs: result output DTOs. These comparisons are done using adapted distance calculations and can then give a quantitative assessment of the TPS error for the tested quality criterion. Results analysis method is developed with the exact knowing of both the input characteristics and the reference algorithm. The use of digital test object for TPS quality control has been validated in previous works. ${ }^{3,5}$

\subsection{Complete DTOs and DTO classes}

The nature of the input and output reference DTOs needs to be the same as the nature of the input and the output of the tool they control. Some DTOs for different tests are based on the same geometry and require the same images slices. Moreover, a DTO that serves as reference output can be used for input in another test. Thus we developed the notion of DTOs. We defined DTO classes that describe the nature of the single DTO and we define the concept of complete DTO. A complete DTO is based on a particular geometry and contains every single DTOs of each class, related to this geometry. The four DTO classes follow the nature of the data used by a TPS:

- images DTOs consist of the CT-slices of the whole DTO, and describe the discrete 3D scene of the DTO;

- structure DTOs contain information about specific contouring descriptions; 
- isocenter DTOs represent an isocenter definition;

- collimator DTOs describe the parameters to conform the treatment machine collimator.

All the DTO classes can be import or export in a DICOM or DICOM-RT file. All the DTO can be generated by DTO-Creator (cf. section 33).

A distance calculation algorithm was defined for each DTO class to compare automatically two objects of same nature. Thus the automatic and quantitative result analysis method of a quality control test is the distance calculation algorithm of the test output DTO class. The structure DTOs are evaluated comparing its volume, center of gravity and roughness with the reference DTO. Isocenter DTOs are evaluated by computing the distance between the two points.

All the DTO class evaluation parameters are computed from a dedicated software that we implemented, from the DICOM files containing the complete DTO including the result output DTOs. Collimator DTOs are compared by studying the position of each leaf, and the irradiated surface area at the isocenter level. We did not define an evaluation method for images DTO class because it is never used as output DTO.

\subsection{Links and chain quality control}

The geometrical treatment planning process is composed by a series (chain) of basic operations (links) computed by the TPS tools. In this chain, the output of a tool is the input of the next one. Each link must be controlled individually as well as the global and partial chains to study the possible errors propagation. With the standard methods, the use of PTOs does not make possible the quality control of a single link but only of the global and the partial chains, including the CT-scanner acquisition. The quality control of one tool is then dependent on the quality of the previous tool in the chain.

For a quality control of the whole chain, only the image part of the DTO is taken for input of the TPS. The physicist computes each step of the beam parameterization following the instructions for the applied test. At the end of this chain, the treatment planning system gives a beam specification that defined the volume that will be irradiated. The quality control consists then to use this information and the reference beam parameters to compare the theoretical irradiated volume to the one defined by the TPS. Figure 1 shows how the quality assessment is organized. The quality control of each link of the chain is described as well as the quality control of the complete and partial chains. In each case, inputs, reference and resulting outputs and the quantitative quality assessment methods are described.

It is moreover possible to individually test each link of this parameterization chain. The first one is the automatic contouring of the volume. The input of the TPS is then only the geometrical part of the test object. The result of the automatic shape contouring by the TPS is compared to the structure of the theoretical contouring, meaning the analytic description of the tested shape. For the auto-margin test, the input test object is the reference output test object of the first link of the chain, in order to have the structure that exactly fits the tested shape of the geometric test object. The expansion of the ROI is done on the TPS following the quality test requirements. The result of this manipulation is compared with the description of the theoretical volume expansion by the specified margins in each spatial direction. For some tests as the analysis of the slice thickness taking into account a three dimensional expansion, several methods can be used and in this case several references can be computed. The TPS result is thus described in function of theses reference structures in order to characterize the TPS expansion algorithm. All of these reference structures of the expansion test are taken as input for the isocenter calculation test. The physicist can then ask the TPS to compute the isocenter coordinates. The beam information files are then used for the reference result since containing the theoretical isocenter position for a given ROI. Moreover the same input can be used for the collimator conformation test. In the same way, the test object beam information can be used as the reference output. The chain of the beam parameterization can now completely be tested. In this purpose, the input of the TPS is the same as the first link test input DTO, and the reference output the same as the reference output DTO of the last tested link. 


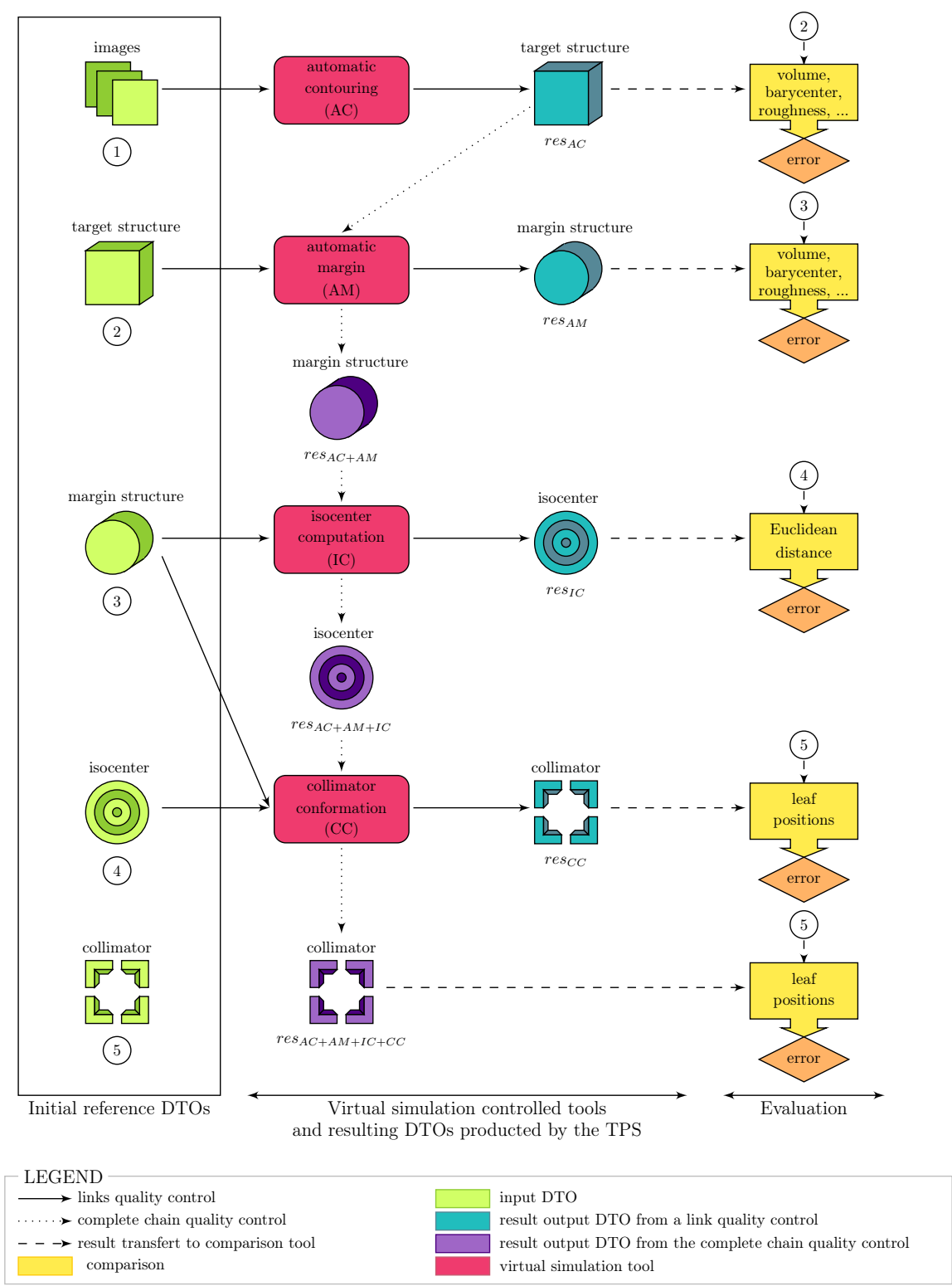

Figure 1. General diagram of the quality control process.

\section{DIGITAL TEST OBJECTS FOR TPS QUALITY CONTROL}

In order to test the concerned TPS tools, several complete DTOs have been designed. Their conception allows for controlling the four geometric tools, testing each of their specificities:

1. auto-contouring: thresholding sensibility, shape contouring and contouring of a branched shape;

2. auto-margin: positive and negative uniform and non-uniform expansions and organ avoidance reaction;

3. isocenter computation: test of the different algorithms in concave and convex major contour shapes;

4. collimator conformation: symmetrical, asymmetrical and multi-leaf collimators.

The eight complete DTOs (i.e. all input and reference output DTOs for a specific geometrical case) have been generated with the help of the DTO generator software: DTO-Creator. At their creation, they get the reference inputs and outputs for each quality test. 


\subsection{Description of the designed DTO shapes}

Eight 3D scenes have been designed to produce the different complete digital test objects defined for the quality control of the geometric tool of the TPS. They have been developed following and adapting the work by Georges Madelis. ${ }^{11}$ The defined DTOs are the following:

- cube: cube of $5 \mathrm{~cm}$ side, cf. figure $2(\mathrm{a})$

- cylinder: cylinder of $5 \mathrm{~cm}$ long and a with a diameter of $5 \mathrm{~cm}$, cf. figure 2(b)

- $U$ : volume with concave shape. The volume is $5 \mathrm{~cm}$ long with a square section of $9 \mathrm{~cm}$ side, and has a parallelepipedic cavity of $3 \mathrm{~cm}$ large and $4 \mathrm{~cm}$ depth, cf. figure 2(c).

- mondrian: volume made of five slices of $1 \mathrm{~cm}$ thick. Each slice has a square shape with variable side dimensions $(5 \mathrm{~cm}, 10 \mathrm{~cm}, 1 \mathrm{~cm}, 5 \mathrm{~cm}$ and $10 \mathrm{~cm})$, cf. figure $2(\mathrm{~d})$

- sphere: sphere with a diameter of $3 \mathrm{~cm}$, cf. figure $2(\mathrm{~g})$.

- double: 3D scene made of two parallelepipedic volumes of $5 \mathrm{~cm}$ thick, and a square section of $4 \mathrm{~cm}$ side. The two volumes are separated by a distance of $2 \mathrm{~cm}$, cf. figure 2(e)

- triple: 3D scene made of three identical parallelepipedic volumes $\left(4 \times 4 \times 2 \mathrm{~cm}^{3}\right)$ aligned two by two according to lateral and head-foot axes and separated by $1 \mathrm{~cm}$, cf. figure $2(\mathrm{f})$

- $M L C$ : divergent volume with a teethed section reproducing the geometry of a multi-leaf collimator. The volume presents a hole at its center used for classical collimator quality test, cf. figure 2(h).

Every DTOs contain shapes with water density (0 HU), in a environment of air density (-1000 HU), execpt for the DTO triple that contains three volumes which have a density of $80 \mathrm{HU}, 90 \mathrm{HU}$ and $95 \mathrm{HU}$ in an environment of $-1000 \mathrm{HU}$. Furthermore, every DTOs have a slice thickness of $1 \mathrm{~cm}$, except for the DTO sphere wich have a slice thickness of $1 \mathrm{~mm}$, all computed without separation between slices. Only the images part of the DTO are presented in this section. Structure, isocenter and collimator DTOs associated to these image DTOs are described in the next section.

\subsection{Quality control process}

We developed some specific tests procedures for each of the four geometric tools previously cited in section 5. The tests are based on the DTOs described in the previous section. The quantitative quality assessment is done with the result analysis method associated to each DTO class.

\subsubsection{Auto-contouring}

The auto-contouring tool is firstly evaluated on its sensibility to the contrast difference between the volume to delineate and the background. In this purpose, we use the DTO triple that presents contrasts of 20 HU, $10 \mathrm{HU}$ and $5 \mathrm{HU}$. The test procedure consists on asking to the TPS to individually contour each of the three volumes of the DTO to evaluate the three proposed situations. The input DTO is thus the images DTO of the complete DTO and the reference output DTOs are three structure DTOs that delineate exactly the three individual shapes.

Then, we propose to evaluate the capacity of this tool to delineate different shapes as cube, cylinder, U, mondrian and sphere. The cube is for plan and angle testing, cylinder and sphere are for rounded shape testing, $U$ is for concave shape testing and mondrian is for testing how the slice thickness is taken into account during the delineation. In the same way, the input DTOs are the images DTOs class of the complete DTO and the reference output are some structure DTOs that delineates exactly all the particular shapes.

To conclude, we finish with a test to evaluate the capacity of the auto-contouring tool to delineate branched shapes. We then use the DTO double to see if the TPS can delineate the two blocks in one structure. The input DTO is the images DTO and a structure DTO that describe the two volumes in a single entity. 


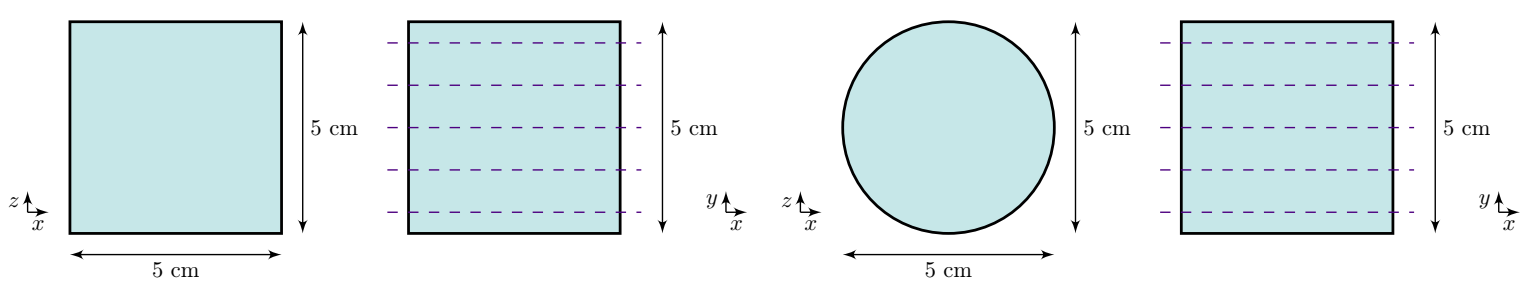

(a) Views of the volume of DTO cube.

(b) Views of the volume of DTO cylinder.

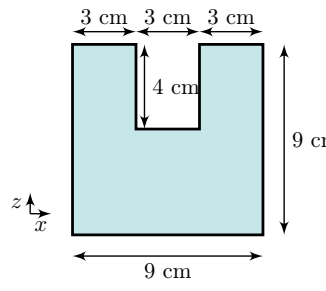

(c) of the volume of DTO $U$.

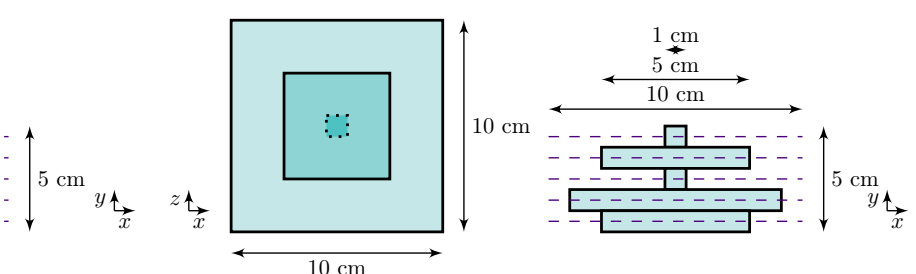

(d) Views of the volume of DTO mondrian.

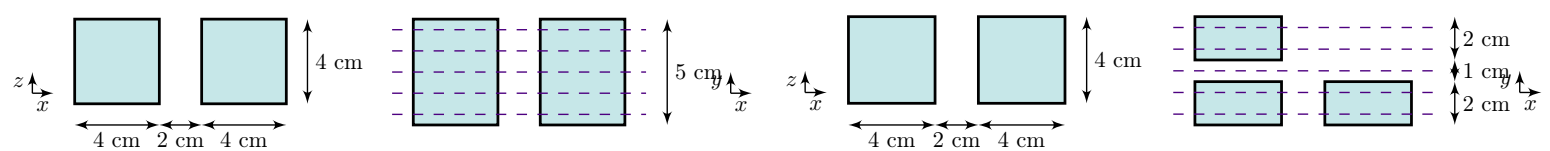

(e) Views of the volume of DTO double.

(f) Views of the volume of DTO triple.

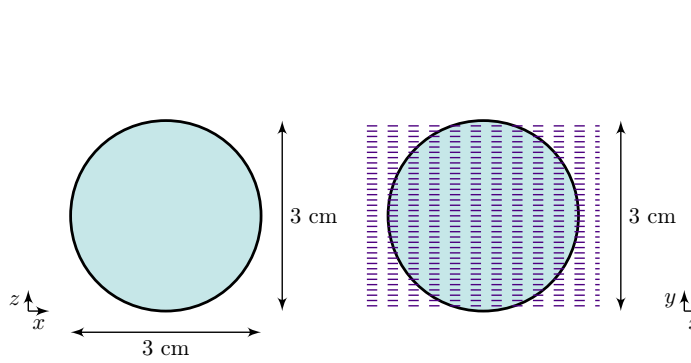

(g) Views of the volume of DTO sphere.

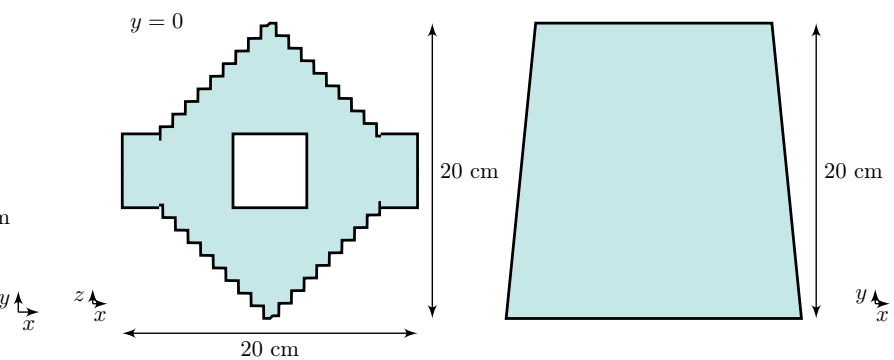

(h) Views of the volume of OTN $M L C$.

Figure 2. Diagrams of the DTO used of geometric TPS tools quality control. The volumes are sliced along the $Y$ axis.

\subsubsection{Auto-margin}

We propose nine test procedures to evaluate the quality of the auto-margin TPS tool. The first eight procedures are carried out with the DTOs cube, cylinder and $U$ to test the positive and negative margins as well as the uniform and non uniform margins. Indeed, a margin is considerate as uniform if it has the same dimensions in the six directions of the 3D space. The input DTOs of these tests are the images DTOs on which the TPS has to proceed the following expansions or erosion:

1. positive and negative uniform margin of $1 \mathrm{~cm}$;

2. positive and negative uniform margin of $2 \mathrm{~cm}$;

3. positive and negative non uniform margin of type 1;

4. positive and negative non uniform margin of type 2 ;

We consider that non uniform margin that conserve the same dimensions for the two directions of a single axis are of type 1 and the other ones are of type 2. If $X$ is the direction for the right of a patient, $Y$ the direction of his head and $Z$ is the top direction, we propose for the type 1, a margin of $1.5 \mathrm{~cm}$ along $X$ axis, $1 \mathrm{~cm}$ along $Y$ 
axis and $0.5 \mathrm{~cm}$ along $Z$ axis. For the non uniform margin of type 2, we propose a margin of $1.5 \mathrm{~cm}$ along $X^{+}$ (positive direction of $X$ axis), $0.5 \mathrm{~cm}$ along $X^{-}$(negative direction of $X$ axis), $2 \mathrm{~cm}$ along $Y^{+}, 1 \mathrm{~cm}$ along $Y^{-}$, $0.5 \mathrm{~cm}$ along $Z^{+}$and $1.5 \mathrm{~cm}$ along $Z^{-}$.

The ninth test controls the TPS capacity to avoid a specific region as healthy organs for instance. The DTO dedicated to this test is the DTO triple. Input DTO is the images part and two structure DTOs that delineate the central volume in one hand, and the two others in a second hand. It is then asked for the TPS to apply a $2 \mathrm{~cm}$ positive margin on the central block, avoiding the other ones, along the slice axis and inside each slice.

Each TPS does not consider the slice thickness in the same way for the auto-margin operation. Some consider the center of the external voxels as the boundary of the shape, and other consider the edge of these voxels. We developed reference output DTOs for the two models as standard to characterize the TPS.

\subsubsection{Isocenter computation}

A TPS usually presents several isocenter positioning algorithms. The isocenter can be placed at the center of gravity of

- the input structure;

- the parallelepiped including the structure;

- the projection of the structure, at a level chosen by the user.

Three isocenter reference DTOs are computed for the DTOs $U$, mondrian and double. The input DTOs belong to the images and structure classes. The structure inputs delineate the shape of each DTO volume.

\subsubsection{Collimator conformation}

We propose to control the conformation of the classical collimators and multi-leaf collimators. The DTO $M L C$ is dedicated to this control. It is basically composed of the images DTO, two structure DTOs (one delineating the whole volume and one delineating the hole in the volume), several isocenter DTOs and the reference output DTOs of each of the proposed tests. Each test consists on conforming the collimator to the input structure using the input isocenter. Output DTOs belong to the collimator class.

To control the symmetric collimators conformation, we use the volume structure DTO and an isocenter DTO positioned at the center of the volume. The output DTOs are the perfect collimator conformation to the structure, with a $0^{\circ}$ or with a $45^{\circ}$ collimator rotation angle. To control the asymmetric collimators conformation, we use the hole structure DTO and an isocenter DTO positioned near a corner of the hole in the volume. The output DTO is the perfect collimator conformation to the hole structure, with a $0^{\circ}$ collimator rotation angle.

The MLC quality control requires several tests to be efficient. The first one consists on conforming the collimator to the teethed volume, with a central isocenter and a non rotated collimator. It allows for checking each leaf positioning. The other tests focus on testing the three conformation algorithms associated to the MLC:

1. all the leaf edges are positioned outside the structure ("outside" algorithm);

2. the centers of the leaf edge are positioned on the structure boundary ("median" algorithm);

3. all the leaf edges are positioned inside the structure ("inside" algorithm).

The tests of these algorithms are also carried out using a central isocenter but conforming the collimator to the hole structure and with a rotation angle of $45^{\circ}$. The reference output collimator DTOs corresponding to the three algorithms are developed and included in the complete DTO. 


\subsubsection{Complete chain quality control}

We also propose a test to study the error propagation in the whole geometrical treatment planning process. We use the DTO sphere. The input DTO is only the images part of the DTO. The reference output collimator DTO is the theoretical result of an auto-contouring, a uniform auto-margin of $1 \mathrm{~cm}$, an isocenter positioning at the barycenter of the structure, following by the conformation of a classical collimator and of an MLC, with a "median" algorithm.

\section{RESULTS}

All the previously presented tests were carried out on the TPS Varian Eclipse (version 7.5.51) at the radiotherapy department of the hospital of La Roche-sur-Yon (France). The four geometric tools have been tested individually as well as the whole geometrical planning chain. The quality control showed an accurate quantitative quality assessment and was very fast. Half a day was enough to proceed the complete quality control. A quality assessment using PTOs takes more time because of the multiple objects positioning and acquisitions.

\subsection{Auto-contouring}

The controlled TPS presented good results for auto-contouring. The sensibility criterion showed some particularities of the TPS in terms of $3 \mathrm{Dl}$ reconstruction of the virtual model.

\subsubsection{Sensibility}

We used two versions of the DTO triple to test this criterion. The first one presented three blocks with densities of $80 \mathrm{HU}, 90 \mathrm{HU}$ and $95 \mathrm{HU}$ in a $100 \mathrm{HU}$ environment, and the second one presented three blocks with densities of $120 \mathrm{HU}, 110 \mathrm{HU}$ and $105 \mathrm{HU}$ in a $100 \mathrm{HU}$ environment too. We compared the auto-contouring of each block with the corresponding reference output DTO. The differences mainly lied in the volume of the resulted structures. Despite the binary values of the DTO densities, the resulted structure depends on the threshold chosen by the operator. The nearer the threshold from the density of the block, smaller the delimited volume. This shows that the TPS 3D reconstruction is done using interpolations. Nevertheless, the center of gravity and the roughness of the result DTOs are similar to the reference DTOs.

\subsubsection{Shape contouring}

Shape contouring did not show significant errors. It was performed on the DTOs cube, cylinder, $U$ and mondrian. Volume errors were inferior to $1 \%$ for the cube, cylinder and mondrian, and centers of gravity were at the same position. The concave shape was delineated with a volume $2 \%$ inferior to the reference. Roughness was the same as the reference DTOs for the cube, $U$ and mondrian. However, the roughness of the cylinder was $6.5 \%$ superior to the one of the reference output DTO. That reflects the TPS difficulties to delineate a rounded shape.

\subsubsection{Branched shape}

The branched shape contouring was tested with the DTO double. Eclipse proceeded well to this exercise. Every result parameters were the same as the ones of the reference output DTO.

\subsection{Auto-margin}

The uniform and non uniform auto-margins were performed on the DTOs cube, cylinder and $U$. The avoidance test were performed on the DTO triple. Two reference output DTOs were computed for each test as we defined them for the two slice thickness interpretation modes. During the quality control of Eclipse, we observed less difference between the DTOs that take the slice thickness into account and the resulting DTO than between the DTOs that do not take the slice thickness into account and the resulting ones. Consequently, we considered only the first series as standard for the TPS auto-margin tool. 


\subsubsection{Uniform margins}

Positive uniform margins seem to be well computed by Eclipse. However, volumes are generally smaller in their results than in the references. For a $1 \mathrm{~cm}$ margin, the volumes are $1 \%$ to $2 \%$ smaller, and for a $2 \mathrm{~cm}$ margin, the volumes are about $3 \%$ smaller. Centers of gravity are respected but the resulted structures are rougher (from $0.5 \%$ to $2.5 \%$ more). The $2 \mathrm{~cm}$ margin fills the cavity of the concave DTO as it was planned.

No difference was found between results and references for the $1 \mathrm{~cm}$ negative uniform margin. However, the $2 \mathrm{~cm}$ negative uniform margin test showed a volume error of $7 \%$ smaller for the DTO cylinder that highlights some erosion algorithm defaults. Moreover, the branch parts of the concave shape disappear with this margin making an irregular contour in the TPS result DTO. That imply a volume $15 \%$ bigger in the Eclipse result.

\subsubsection{Non uniform margins}

The tests of non uniform auto-margin of type 1 and 2 showed significant differences with the reference results specially for the application to the DTO cylinder. It highlighted the shape of the structural element of the TPS for non uniform margin. Indeed, the resulted structures were more angular, bigger for a negative margin (18\% bigger for type 1) and smaller for a positive margin (2\% smaller for type 1$)$. The differences between the positive and the negative errors are due to the relative error quantification. The application of these types of margin showed differences with our standard on the $U$ shape. The margin dimensions are underestimated. The result is that the positive margin does not fill the cavity as awaited and the branch did not disappear as in the theoretical result. The figure 3 show the result of the negative non uniform auto-margin of type 1 on the cylinder and the $U$.

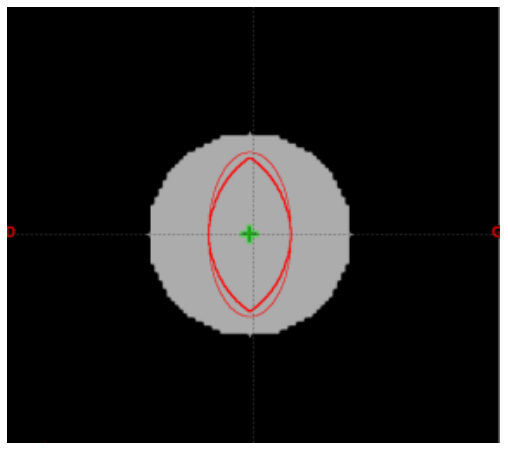

(a) Cylinder.

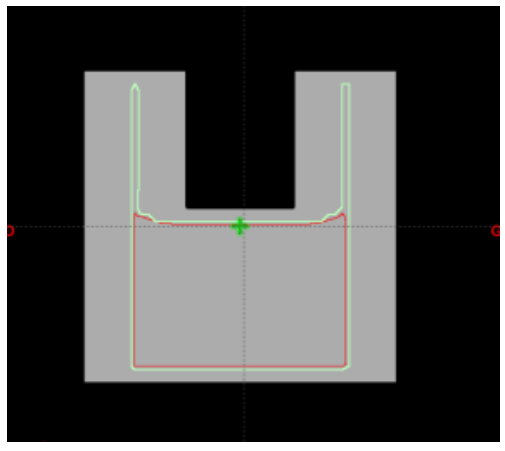

(b) Concave shape.

Figure 3. Reference and result (in thick lines) output DTO for the negative non uniform auto-margin of type 1.

\subsubsection{Structure avoidance}

Two types of avoidance have been tested with the DTO triple. The first one consists on avoiding a structure in the same slice than the one to expand. The second one consists on avoiding a structure along the slices axis. The automatic quantitative result analysis of these tests concluded that the result output DTO is $8 \%$ smaller than the reference one and 13\% rougher (cf. figure 44). However, these tests should require another result analysis algorithm in order to check the avoidance criterion.

\subsection{Isocenter computation}

Eclipse offers two isocenter positioning options. Isocenter can placed the center of gravity of the structure, or at the level of the center of gravity of the projection of the structure from the beam source. We tested these two options with the DTO $U$ including the two reference output DTOs. The errors are taken along each axis and the maximal found was about $0.4 \mathrm{~mm}$ for the positioning at the center of gravity of the structure. 


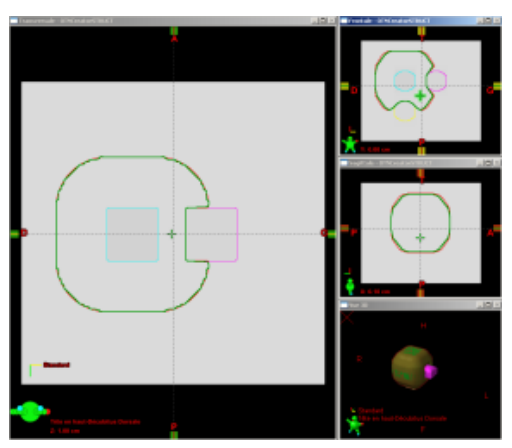

(a)

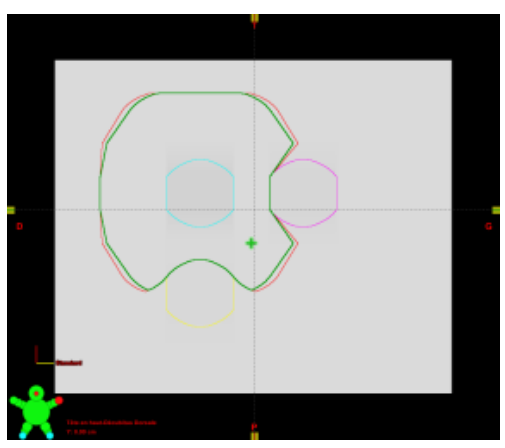

(b)

Figure 4. Avoidance test result with Eclipse (reference output DTO in thin lines and result output DTO in thick lines).

\subsection{Collimator conformation}

We tested the Eclipse tool for collimator conformation with the dedicated DTO $M L C$, for classical and multi-leaf collimators. The collimator result DTO of classical collimator test was analyzed with the appropriate quantitative method and no position error was found for symmetrical and asymmetrical collimator conformation.

For the MLC conformation verification, we firstly used the teethed shape, without rotation angle, and then we tested the three conformation algorithms. The mean leaf position error was about $0.2 \mathrm{~mm}$ but the maximal error was about $2 \mathrm{~mm}$ for all the tests. However we could remark studying the displays and the detailed result analysis that some leafs present an excessive closing.

\subsection{Complete chain quality control}

The complete beam parametrization process was tested with the DTO sphere. We proceeded to an autocontouring, an auto-margin of $1 \mathrm{~cm}$ and a classical symmetric conformation as well as an MLC conformation. The result output DTOs were compared to the references with the automatic collimator DTO quantitative analysis method. We could thus study the error propagation in the whole chain. Very few errors were found on the classical collimator and MLC conformation. Theoretical and resulting irradiated surfaces differed from less than $1 \mathrm{~mm}^{2}$. Thus, we can conclude that the Eclipse beam parametrization process quality is satisfying for the tested conditions.

\section{DISCUSSION}

The major particularity of the digital test objects is that they do not need to be acquired by a CT-scanner operation. This insures a perfect positioning of the shapes in the volume that is not guaranteed by the use of a physical test object due to possible errors on the object alignment on the CT-scanner table. Moreover, the CT-scanner acquisition introduces blur, noise and spatial distortions in the digital 3D representation of the phantom. This perfect positioning and non deteriorated object contribute to a real quality assessment because biases are not introduced at the TPS test input.

A physical test object like the Modus Quasar phantom allows to test the whole beam parametrization chain but not each individual link because of its time-consuming CT-scanner acquisition. Indeed, for the auto-margin test for instance, it is necessary to first compute a targeting in order to obtain the volume to expand. If the TPS presents some error in its targeting tools, then it will be difficult to isolate the expansion ones from them. Thus, the expansion error assessment will depends on the targeting performance of the treatment planning system.

Digital test objects are complete objects. Contrary to the physical ones, they contain structures and beams information. Thus, it is possible to have input and output references for each test. The results of the TPS can be compared to the theoretical response for the given quality test. This leads to the opportunity to define an automatic result analysis method in order to quantify the treatment planning system errors. ${ }^{13}$ It is moreover easy to display the theoretical result and the real one to visualize the differences. 
Only the Digital Test Objects allow a complete quality control for treatment planning systems. Moreover DTOs are in all situations more accurate than physical test objects. A fully automatic quality assessment is obtained thanks to the objects digital nature and leads to a less time-consuming quality control process as they do not need a CT-scanner acquisition.

\section{CONCLUSION}

A new quantitative quality assessment method for geometrical treatment planning tools has been developed. This method, based on the use of digital test objects, allows an accurate control of each individual tool without being dependent on the quality of the other tools. The quality assessment is automatic and quantitative. This technique leads to a fast quality control with a precise and complete error computation. Some test procedures have been defined but it is easy to establish new one just adding new DTOs to the complete one. Our methodology allows to control each tool individually as well as the entire or partial beam parametrization procedure.

\section{REFERENCES}

1. T. Craig, D. Brochu, and J. Van Dyk, "A quality assurance phantom for three-dimensional radiation treatment planning," Int. J. Radiat. Oncol. Biol. Phys. 44(4), pp. 955-966, 1999.

2. J.-N. Foulquier, "Contrôle de qualité d'une installation de simulaiton virtuelle. les outils de le simulation virtuelle," tech. rep., Hôpital Tenon, service de radiothérapie, unité de physique médicale, Paris, 2002.

3. E. Denis, S. Beaumont, and J.-P. Guédon, "Digital reconstructed radiography quality control with software methods," in Proceedings of SPIE, Medical Imaging 2005: Physics of medical imaging, M. Flynn, ed., 5745, pp. 1002-1013, SPIE, 2005.

4. E. Denis, J.-P. Guédon, S. Beaumont, and N. Normand, "Discrete and continuous description of a three dimensional scene for quality control of radiotherapy treatment planning systems," in Proceedings of SPIE, Medical Imaging 2006: Physics of medical imaging, M. Flynn, ed., 6142, SPIE, 2006.

5. E. Denis, S. Beaumont, J.-P. Guédon, N. Normand, and T. Torfeh, "Automatic quality control of digitally reconstructed radiograph computation and comparison with standard methods," in Proceedings of SPIE, Medical Imaging 2007: Physics of medical imaging, M. Flynn, ed., 6510, SPIE, 2007.

6. B. Fraas, K. Doppke, M. Hunt, G. Kutcher, G. Starkschall, and J. Stern, R. Van Dyke, "American Association of Physicists in Medicine Radiation Therapy Committee Task Group 53: Quality assurance for clinical radiotherapy treatment planning," Med. Phys. 25, pp. 1773-1829, October 1998.

7. S. Mutic, J. Palta, E. Butker, I. Das, M. Huq, L.-N. Dick Loo, B. Salter, C. McCollough, and J. Van Dyk, "Quality assurance for computed-tomography simulators and the computed-tomography-simulation process: Report of the AAPM Radiation Therapy Committee TG No.66," Med. Phys. 30(10), pp. 2762-2792, 2003.

8. P. Andreo, M. Cohen, J. Gultresa, J. Haywood, J. Novotny, M. Oresegun, P. Ortiz-López, D. Seng, M. Stovall, J. van de Geijn, J. Vivanco, and P. Zúg̃niga Bello, "Lessons learned from accidental exposures in radiotherapy," Safety Reports Series Nř17, International Atomic Energy Agency (IAEA), 2000.

9. P. Andreo, J. Cramb, B. Fraass, F. Ionescu-Farca, J. Izewska, V. Levin, B. Mijnheer, J.-C. Rosenwald, P. Scalliet, K. Shortt, J. Van Dyk, and S. Vatnitsky, "Commissioning and quality assurance of computerized planning systems for radiation treatment of cancer," Tech. Rep. 430, IAEA, 2004.

10. Modus, "Quality assurance system for advanced radiotherapy," Phys. Med. Biol. 47(9), pp. 1485-1492, 2002.

11. G. Madelis, Assurance qualité et analyse des incertitudes en radiotherapie conformationnelle pour le cancer de la prostate. PhD thesis, Université de Toulouse 3, Toulouse, France, 1999.

12. NEMA, "Digital Imaging and Communications in Medicine (DICOM)," tech. rep., National Electrical Manufacturers Association, 2007.

13. QualiFormeD. http://www.qualiformed.com. 